\title{
ORLICZ DUAL LOGARITHMIC MINKOWKI INEQUALITY
}

\author{
CHANG-JIAN ZHAO
}

Abstract. In this paper, we establish an Orlicz dual logarithmic Minkowski inequality by introducing a new concept of Orlicz dual mixed volume measure, and using the newly established Orlicz dual Minkowski inequality. The Orlicz dual logarithmic Minkowski inequality in special case yields the dual logarithmic Minkowski inequality. The $L_{p}$-dual mixed volume measure and $L_{p}$-dual logarithmic Minkowski inequality are first derived here.

Mathematics subject classification (2020): 46E30, 46A20, 52A20, 52A30.

Keywords and phrases: Dual mixed volume, Orlicz dual mixed volume, dual logarithmic Minkowski inequality, Orlicz dual Minkowski inequality.

\section{REFERENCES}

[1] K. J. BÖRÖCZKY, E. LutwaK, D. YANG, G. Zhang, The log-Brunn-Minkowski inequality, Adv. Math 231, (2012), 1974-1997.

[2] Y. D. Burago, V. A. Zalgaller, Geometric Inequalities, Springer-Verlag, Berlin, 1988.

[3] A. Coles anti, P. Cuoghi, The Brunn-Minkowski inequality for the n-dimensional logarithmic capacity of convex bodies, Potential Math 22, (2005), 289-304.

[4] M. FAthi, B. Nelson, Free Stein kernels and an improvement of the free logarithmic Sobolev inequality, Adv. Math 317, (2017), 193-223.

[5] W. J. FiREY, Polar means of convex bodies and a dual to the Brunn-Minkowski theorem, Canad. J. Math 13, (1961), 444-453.

[6] R. J. Gardner, D. Hug, W. Weil, Operations between sets in geometry, J. Eur. Math. Soc 15, 6 (2013), 2297-2352.

[7] M. Henk, H. Pollehn, On the log-Minkowski inequality for simplices and parallelepipeds, Acta Math. Hungarica 155, (2018), 141-157.

[8] S. Hou, J. XIAO, A mixed volumetry for the anisotropic logarithmic potential, J. Geom Anal 28, (2018), 2018-2049.

[9] C. LI, W. WANG, Log-Minkowski inequalities for the $L_{p}$-mixed quermassintegrals, J. Inequal. Appl 2019, (2019): 85.

[10] E. LutwaK, Centroid bodies and dual mixed volumes, Proc. London Math. Soc 60, 3 (1990), $365-$ 391.

[11] E. LutwaK, Dual mixed volumes, Pacific J. Math 58, (1975), 531-538.

[12] E. LutwaK, Intersection bodies and dual mixed volumes, Adv. Math 71, (1988), 232-261.

[13] E. LutwaK, The Brunn-Minkowski-Firey theory. II. Affine and geominimal surface areas, Adv. Math 118, (1996), 244-294.

[14] S. LV, The $\varphi$-Brunn-Minkowski inequality, Acta Math. Hungarica 156, (2018), 226-239.

[15] L. MA, A new proof of the log-Brunn-Minkowski inequality, Geom. Dedicata 177, (2015), 75-82.

[16] C. Saroglou, Remarks on the conjectured log-Brunn-Minkowski inequality, Geom. Dedicata 177, (2015), 353-365.

[17] R. SchneIder, Convex Bodies: The Brunn-Minkowski Theory, Cambridge University Press, 2014.

[18] A. StAnCU, The logarithmic Minkowski inequality for non-symmetric convex bodies, Adv. Appl. Math 73, (2016), 43-58.

[19] W. Wang, M. Feng, The log-Minkowski inequalities for quermassintegrals, J. Math. Inequal 11, (2017), 983-995. 
[20] W. WANG, G. Leng, $L_{p}$-dual mixed quermassintegrals, Indian J. Pure Appl. Math 36, (2005), 177188.

[21] W. WANG, L. LiU, The dual log-Brunn-Minkowski inequalities, Taiwanese J. Math 20, (2016), 909919.

[22] X. WAnG, W. Xu, J. Zhou, Some logarithmic Minkowski inequalities for nonsymmetric convex bodies, Sci. China, 60, (2017), 1857-1872.

[23] C.-J. ZHAO, The dual logarithmic Aleksandrov-Fenchel inequality, Balkan J. Geom. Appl 25, (2020), $157-169$.

[24] C.-J. Zhao, The log-Aleksandrov-Fenchel inequality, Mediterr. J. Math (2020), 17: 96.

[25] C.-J. ZHAO, Orlicz log-Minkowski inequality, Diff. Geom. Appl 74, (2021): 101695.

[26] C.-J. ZHAO, The areas log-Minkowski inequality, Revista de la Real Academia de Ciencias Exactas, Físicas y Naturales. Serie A. Matemáticas 115, (2021): 131.

[27] B. Zhu, J. Zhou, W. Xu, Dual Orlicz-Brunn-Minkwski theory, Adv. Math 264, (2014), 700-725. 